\title{
Research on hydraulic system of hydraulic elevator

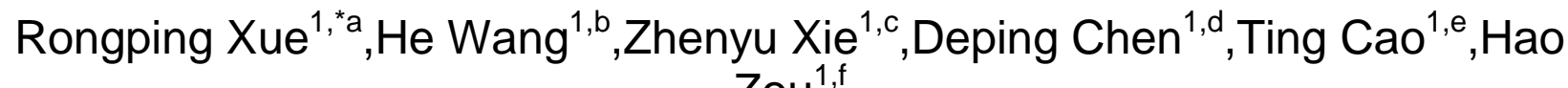 $\mathrm{Zou}^{1, \mathrm{f}}$
}

${ }^{1}$ Sichuan special equipment inspection and Research Institute, Chengdu 610061, China

*Corresponding author

Email:a937373532@qq.com, ${ }^{b}$ Wanghe197656@163.com, ${ }^{\mathrm{c} 973797167 @ q q . c o m,{ }^{\mathrm{d}} 465765960 @ q q .}$ com, ${ }^{\circledR} 53175803 @ q q . c o m$ fsczouhao@163.com

Keywords: the hydraulic system, the hydraulic elevator, the bidirectional speed detection method, car.

Abstract. The purpose of this study is to provide an improved design for the hydraulic system of a certain type of hydraulic elevator in the elevator test. This paper is mainly through the speed feedback control scheme to achieve real-time speed correction, adopted the speed bidirectional detection method to establish the relationship between the car, velocity, acceleration and its corresponding hydraulic cylinder displacement, velocity and acceleration. And the simulation results show that the improved scheme has better dynamic response.

\section{Introduction}

With the rapid development of economy and large number of construction of city high-rise buildings, the elevators have become an indispensable vertical transportation tool in people's life and its technology has also been considerable development. Hydraulic technology as a conventional electromechanical hydraulic integration technology, to warding the green-friendly energy-saving development. High power quality ratio enables it to achieve stepless speed regulation and overload protection. What's more, the hydraulic elevator with flow-electric feedback control has the advantages of safety, reliability, simple structure, low noise, smooth running, comfortable ride, convenient maintenance and no pollution, so that it's loved by the people and very broad prospects for application.

\section{The characteristics of the hydraulic elevator}

The hydraulic elevator is the product of electromechanical hydraulic integration, which rely on the hydraulic power source to drive the hydraulic oil into the oil cylinder to make the plunger for linear motion, and directly or indirectly through the wire rope to make the capsule move. It's mainly composed of pumping station system, hydraulic system, guiding system, car, door system, electric control system, safety protection system and so on ${ }^{[1]}$.

The hydraulic elevator is equipped with a traction type elevator safety devices and a overflow valve to prevent the system pressure is too high when the upward movement. Power supply failure, the emergency manual valve can make the car emergency position down to the nearest floor. When the hydraulic system pipeline rupture car stall drops, the pipeline rupture valve can automatically cut off the oil. When the oil temperature in the fuel tank exceeds a certain threshold, the oil temperature protection device sends a signal to suspend the elevators, and after the temperature drops can restart the elevator. Using low-noise screw pumps, pumps and motors are designed into a submersible structure, which forms a pump seal overall, and it has the effect of explosion-proof, flame retardant, and noise reduction. When down, the hydraulic elevator relies on the pressure generated by the pressure driven can save energy. Foreign statistics show that the total cost of hydraulic elevators can be cheaper than traction elevators for buildings $40 \mathrm{~m}$ below. 


\section{Inspection of a type hydraulic system of hydraulic elevator}

The existing hydraulic elevator generally have the cylinder and the hydraulic pump station which connected with the cylinder, the solenoid directional valve is connected to the hydraulic cylinder through the oil circuit. When the oil pump injected hydraulic oil into the hydraulic cylinder, the elevator car upstream and the solenoid directional valve unloading elevator car downstream. This elevator has the advantages of simple configuration, low cost, safety and reliability. But the reason for the system itself, the hydraulic rod extended contraction speed is not smooth, and there is a deviation, so that the car does not run smooth.

The following Fig.1 shows the schematic diagram of a certain type of hydraulic elevator speed feedback control of the hydraulic system during the inspection, the system consists of two parts, the hydraulic system and the electro-hydraulic control system.

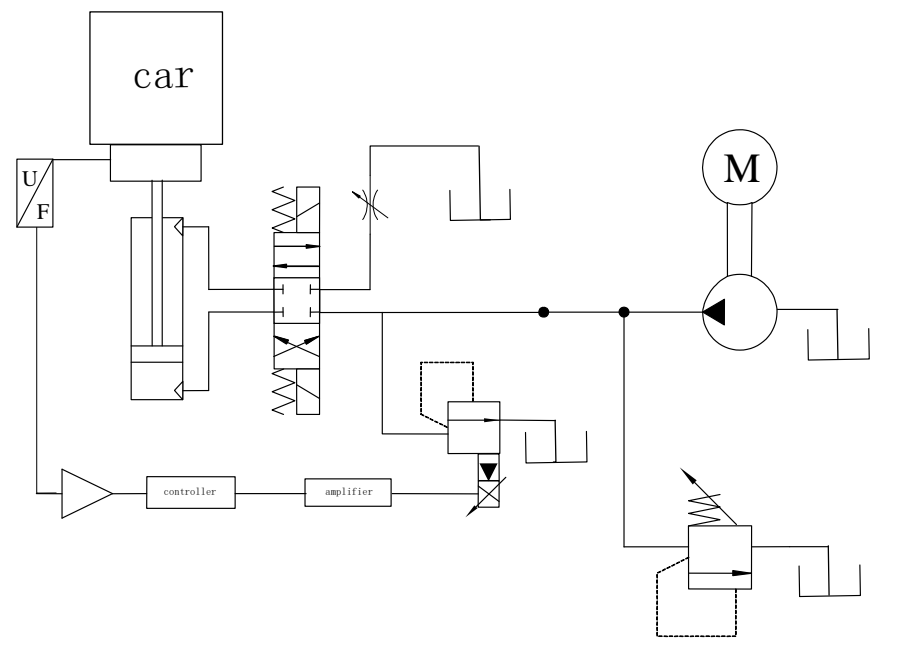

Fig. 1 Inspection of the elevator hydraulic schematic diagram

As shown in Figure 1, the hydraulic circuit is composed of the quantitative pump, the relief valve, the proportional relief valve, the solenoid directional valve, the hydraulic cylinder, the position sensor, the throttle valve, the amplifier, fuel tank, motor and so on.

\section{The hydraulic system.}

The relief valve set the oil pressure of the hydraulic system to ensure the oil pressure constant after running. When the electro-hydraulic proportional of the relief valve receives the electrical signal, by changing the position of the valve, thereby changing the output pressure of the relief valve to adjust the inlet pressure of the solenoid directional valve, by controlling the output force of the hydraulic cylinder, so as to control the output size of the force to the car by the hydraulic cylinder. Using the pressure-flow gain of the solenoid directional valve to increase the damping ratio of the system and improve the stability of the hydraulic system. The throttle valve is mainly used to control the traffic of the reflux way to ensure the smooth operation of the hydraulic cylinder $^{[2]}$.

\section{The electro-hydraulic control system.}

The position sensor detects the displacement of the capsule, the electrical signal inputs the amplifier after through the controller. After amplified by the power amplifier to drive the proportional solenoid, so as to control the valve opening of the relief valve.

\section{The improved hydraulic system of the hydraulic elevator}

For the deficiency of the hydraulic system, this paper presents a new hydraulic system. It can real-time monitor the upstream and downstream speed deviation of the compensation capsule, so that the speed change of the hydraulic rod is more slowly and steadily and the capsule run more smoothly, safe and reliable. The improved hydraulic system of the hydraulic elevator is as following: 


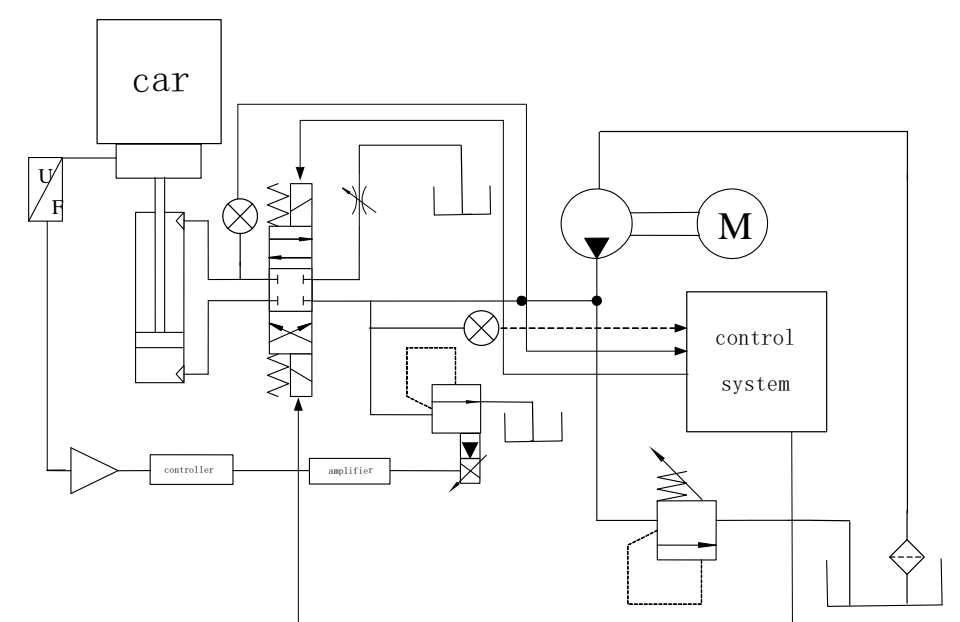

Fig.2 The improved hydraulic system of the hydraulic elevator

This system is consists of the oil circuit system, the control system and the electro-hydraulic proportional valve system. The hydraulic oil in the oil tank is driven by the hydraulic pump station, flowing into the solenoid directional valve and the electro-hydraulic proportional valve system, then into the bottom of the hydraulic cylinder to drive the hydraulic rod extended, and the lift up. Above the process, the speed detection system real-time monitoring, when the velocity changes is not reliable, the speed monitoring system transmits signals to the amplifier, and the amplifier transmits signals to the electro-hydraulic proportional valve system, then the electro-hydraulic proportional valve spool action to adjust the size of the opening and the flow of the hydraulic oil, compensating the operation deviation of the speed, so that the speed change is small and the car run smoothly ${ }^{[3]}$.

The solenoid directional valve connects the upstream and downstream oil pressure detector to monitor of upstream and downstream oil pressure condition in real time, then feedback the monitoring values to the control system and the control system to make feedback, then real-time adjusts the speed of the hydraulic pump, by adjusting the displacement of the hydraulic pump to adjust the speed, so as to compensate for the speed deviation and make the car speed run more smoothly ${ }^{[4]}$.

\section{Dynamic response characteristics of the system}

The mathematical models of two kinds of hydraulic systems are established respectively, to obtain the transfer function of the two systems. Substitution of the relevant data, then getting two transfer function value of the two systems. The step dynamic response curves of the two system by computer simulation are seen in Fig 3 (A) and (B).

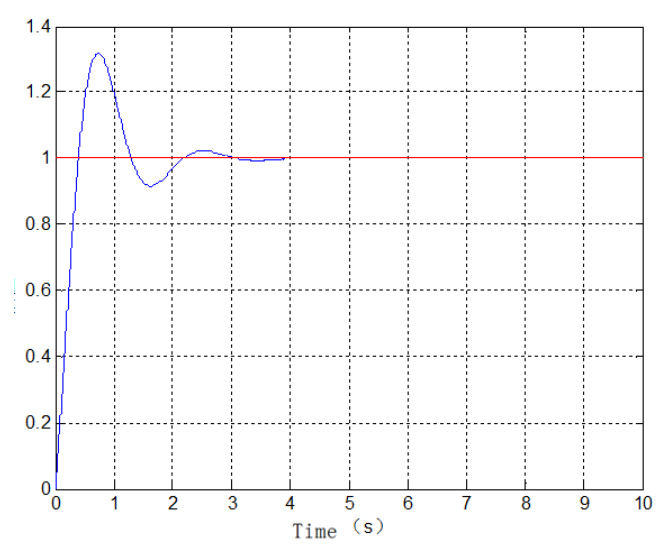

(A)

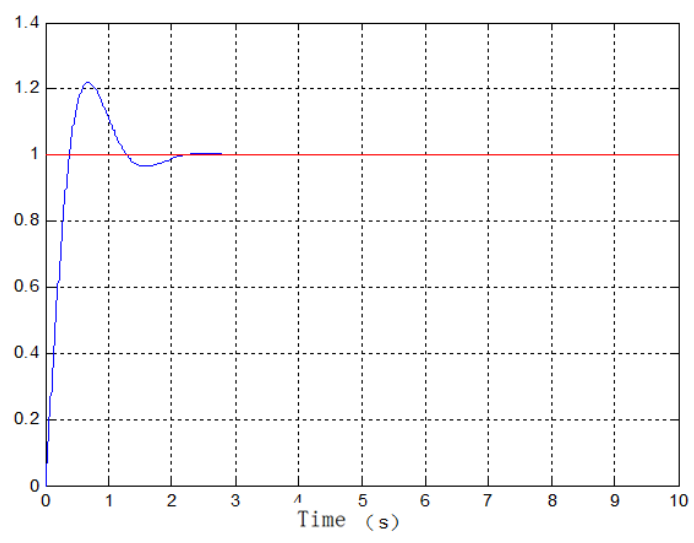

(B)

Fig 3 The step dynamic response curves of the two system 


\section{Summary}

The simulation results show that the improved hydraulic system can be more stable. With the speed bidirectional detection function of the hydraulic system runs more smoothly and rides more comfortable and secure.

\section{References}

[1]. Yongxiang Lu. Electro-hydraulic proportional control technology (1 Edition)[J]. Beijing Machinery Industry Press, 1988.

[2]. Lizhen Zhang. Research on speed feedback hydraulic elevator operating characteristics[C], 1999.

[3]. Guojun Zhao, Huayong Yang. The hydraulic elevator system with car speed feedback control. Zhejiang University of Technology, Hangzhou, 1996.

[4]. Baichang Li. The electro-hydraulic proportional control and digital control system(2 Edition)[J]. Beijing Machinery Industry Press, 1997. 